Research Article

\title{
On Fuzzy Deductive Systems of Hilbert Algebras
}

\author{
Gezahagne Mulat Addis $(\mathbb{D}$ and Derso Abeje Engidaw \\ Department of Mathematics, University of Gondar, Gondar, Ethiopia \\ Correspondence should be addressed to Gezahagne Mulat Addis; buttu412@yahoo.com
}

Received 28 March 2020; Revised 17 June 2020; Accepted 24 July 2020; Published 1 September 2020

Academic Editor: Mehdi Ghatee

Copyright (c) 2020 Gezahagne Mulat Addis and Derso Abeje Engidaw. This is an open access article distributed under the Creative Commons Attribution License, which permits unrestricted use, distribution, and reproduction in any medium, provided the original work is properly cited.

In this paper, we study fuzzy deductive systems of Hilbert algebras whose truth values are in a complete lattice satisfying the infinite meet distributive law. Several characterizations are obtained for fuzzy deductive systems generated by a fuzzy set. It is also proved that the class of all fuzzy deductive systems of a Hilbert algebra forms an algebraic closure fuzzy set system. Furthermore, we obtain a lattice isomorphism between the class of fuzzy deductive systems and the class of fuzzy congruence relations in the variety of Hilbert algebras.

\section{Introduction}

The pioneering work of Zadeh [1] on fuzzy subsets of a set has been extensively applied to many scientific fields. This concept was adapted by Rosenfeld [2] to define fuzzy subgroups of a group. Since then, many authors have been studying fuzzy subalgebras of several algebraic structures such as rings, modules, vector spaces, lattices, pseudocomplemented semilattice, MS algebras, and universal algebras (see [3-8]). More recently, fuzzy ideals of a poset were studied by Alaba et al. [9] as a generalization of those fuzzy ideals of lattices. However, Swamy and Raju $[10,11]$ have unified all these fuzzy algebraic notions, by introducing the general theory of algebraic fuzzy systems. Some applications of fuzzy algebras and fuzzy points are also given in the literature [12-14].

It was Gougen [15] who first realized that the unit interval $[0,1]$ is insufficient to take truth values for fuzzy statements. Swamy and Swamy [16] have suggested that complete lattices satisfying the infinite meet distributive property are the most suitable candidates to take truth values of the general fuzzy statements.

On the other hand, in 1966, Diego [17] introduced the notion of Hilbert algebras and deductive systems and provided various properties. The theory of Hilbert algebras and deductive systems was further developed by Busneag in a series of papers $[18,19]$. Jun and Hong introduced the notion fuzzy deductive system in a Hilbert algebra in [20], and Jun constructed its extension in [21] whose truth values are in the unit interval $[0,1]$ of real numbers.

Initiated by all the above results, in this paper, we define fuzzy deductive systems of Hilbert algebras whose truth values are in a complete lattice satisfying the infinite meet distributive law and we obtain several equivalent conditions for a fuzzy set to be a fuzzy deductive system. Fuzzy deductive systems generated by a fuzzy subset are characterized in different ways. It is also proved that the set of all fuzzy deductive systems of a Hilbert algebra forms an algebraic closure fuzzy set system with respect to the pointwise ordering. Furthermore, we study fuzzy congruence relations on Hilbert algebras and we obtain a lattice isomorphism between the set of all fuzzy deductive systems and the set of all fuzzy congruence relations of a Hilbert algebra.

\section{Preliminaries}

In this section, we recall some definitions and basic properties of deductive systems of a Hilbert algebra. 
Definition 1. An algebra $(A, *, 1)$ of type $(2,0)$ is said to be a Hilbert algebra if it satisfies the following conditions:

(1) $x *(y * x)=1$,

(2) $(x *(y * z)) *((x * y) *(x * z))=1$,

(3) if $x * y=1=y * x$, then $x=y$,

for all $x, y, z \in H$ and 1 is a fixed element of $H$.

It was proved by Diego in [17] that the class $\mathscr{K}$ of Hilbert algebras forms a variety. The following result is also adopted from [17].

Lemma 1. Every Hilbert algebra A satisfies the following conditions:

(1) $x * x=1$,

(2) $1 * x=x$,

(3) $x * 1=1$,

(4) $x *(y * z)=y *(x * z)$,

(5) $x *(y * z)=(x * y) *(x * z)$,

(6) $(x * y) *((y * z) *(x * z))=1$,

(7) $(x * y) *((z * x) *(z * y))=1$, for any $x, y, z \in A$.

If $A=\{1\}$, then $\mathrm{A}$ is called trivial. The binary relation ' $\leq$ ' defined in a Hilbert algebra $A$ by $x \leq y$ if and only if $x^{*} y=1$ is a partial order on $A$ with the greatest element 1 . This order is called the natural ordering on $A$.

The concept of a deductive system on a Hilbert algebra $A$ was introduced by Diego [17] as follows.

Definition 2. A nonempty subset $D$ of a Hilbert algebra $A$ is called a deductive system on $A$ if it satisfies the following conditions:

(1) $1 \in D$,

(2) $x \in D$,

(3) $x^{*} y \in D$, imply $y \in D$.

In other words, a deductive system of $A$ is a subset of $A$ containing 1 and closed under a deduction. One can easily check that the lattice of deductive systems of a Hilbert algebra $A$ is closed under arbitrary intersection so that for any subset $S$ of $A$, always there exists a smallest deductive system $D(S)$ of $A$ containing $S$. It is called the deductive system of $A$ generated by $S$. Chajda [22] has characterized $D(S)$ in the following way. Define sets

$$
\begin{aligned}
& X_{1}=S \cup\{1\}, \\
& X_{n}=\left\{y \in A: x \in X_{n-1} x^{*} y \in X_{n-1}\right\},
\end{aligned}
$$

for each positive integer $n \geq 2$, inductively. Then,

$$
D(S)=\bigcup\left\{X_{n}: n \in \mathbb{N}\right\},
$$

where $\mathbb{N}$ denotes the set of positive integers.

The internal structure of $D(S)$ is also characterized by Diego in [17] as follows: $D(S)=\{1\}$ if $S=\varnothing$ and for $S \neq \varnothing$,

$$
\begin{aligned}
D(S) & =\left\{y \in A: x_{1} *\left(x_{2} *\left(\cdots *\left(x_{n} * y\right) \cdots\right)\right)\right. \\
& \left.=1 \text { for some } x_{1}, x_{2}, \ldots, x_{n} \in S\right\} .
\end{aligned}
$$

Throughout this paper, $L$ stands for a complete lattice satisfying the infinite meet distributive law and $A$ stands for a Hilbert algebra unless and otherwise stated.

By an $L$-fuzzy subset $\mu$ of $A$, we mean a mapping $\mu: A \longrightarrow L$. The set $\{\mu(x): x \in A\}$ is called the image of $\mu$ and is denoted by $\operatorname{Im}(\mu)$. An $L$-fuzzy subset $\mu$ of $A$ is called normalized or unitary if $1 \in \operatorname{Im}(\mu)$. The class of all $L$-fuzzy subsets of $A$ is denoted by $L^{A}$.

For each $\alpha \in L$, the $\alpha$-level subset of $\mu$, which is denoted by $\mu_{\alpha}$, is a subset of $A$ given by

$$
\mu_{\alpha}=\{x: \mu(x) \geq \alpha\}
$$

For any $L$-fuzzy subset $\mu$ of $A$ and each $x \in A$, it was proved in [23] that

$$
\mu(x)=\vee\{\alpha \in L: \mu(x) \geq \alpha\} .
$$

For $L$-fuzzy subsets $\mu$ and $\sigma$ of $A$, we write $\mu \subseteq \sigma$ to mean $\mu(x) \leq \sigma(x)$ for all $x \in A$ in the ordering of $L$. It can be easily verified that " $\subseteq$ " is a partial order on the set $L^{A}$ and it is called the pointwise ordering.

For each $x$ in $A$ and $0 \neq \alpha$ in $L$, we define $x_{\alpha} \in L^{A}$ as follows:

$$
x_{\alpha}(y)= \begin{cases}\alpha & \text { if } y=x \\ 0 & \text { otherwise }\end{cases}
$$

for each $y \in A$ and we call it an $L$-fuzzy point of $A$.

\section{L-Fuzzy Deductive Systems}

In this section, we define $L$-fuzzy deductive system in a Hilbert algebra $A$ and investigate some of its properties.

Definition 3. An $L$-fuzzy subset $\mu$ of $A$ is said to be an $L$-fuzzy deductive system on $A$ if

(1) $\mu(1)=1$,

(2) $\mu(x) \wedge \mu(x * y) \leq \mu(y)$, for all $x, y \in A$.

We denote by $F D_{L}(A)$ the set of all $L$-fuzzy deductive systems of $A$.

The following lemma is a straightforward verification of the definition, but useful to prove results in the latter section.

Lemma 2. Let $\mu$ be an L-fuzzy deductive system on $A$. Then, for any $x, y, z, w \in A$, the following hold:

(1) $\mu(x * y)=1$ implies $\mu(x) \leq \mu(y)$,

(2) $x \leq y$ implies $\mu(x) \leq \mu(y)$,

(3) $x *(y * z)=1$ implies $\mu(x) \wedge \mu(y) \leq \mu(z)$,

(4) $\mu(x * z) \wedge \mu(z * y) \leq \mu(x * y)$. 
Proof

(1) Let $x, y \in A$ such that $\mu(x * y)=1$. Then, we have the following:

$$
\mu(x)=\mu(x) \wedge 1=\mu(x) \wedge \mu(x * y) \leq \mu(y) .
$$

(2) Let $x, y \in A$ such that $x \leq y$. Then, $x * y=1$ and hence, $\mu(x * y)=\mu(1)=1$. Thus, it follows from (1) that $\mu(x) \leq \mu(y)$.

(3) Let $x, y, z \in A$ such that $x *(y * z)=1$. Then, $x \leq y * z$. By (2), we have $\mu(x) \leq \mu(y * z)$. This implies that

$$
\mu(x) \wedge \mu(y) \leq \mu(y * z) \wedge \mu(y) \leq \mu(z) .
$$

(4) Let $\quad x, y, z \in A$. By Lemma $1, \quad(x * z) *$ $((z * y) *(x * y))=1$. Then, it follows from (3) that $\mu(x * z) \wedge \mu(z * y) \leq \mu(x * y)$.

Lemma 3. An L-fuzzy subset $\mu$ of $A$ is an L-fuzzy deductive system of $A$ if and only if the $\alpha$-level subset $\mu_{\alpha}$ is a deductive system of $A$, for all $\alpha \in L$.

Proof. Let $\mu$ be an $L$-fuzzy deductive system of $A$ and $\alpha \in L$. Then, $\mu(1)=1 \geq \alpha$. Thus, $1 \in \mu_{\alpha}$. Again, let $x, y \in A$ such that $x \in \mu_{\alpha}$ and $x * y \in \mu_{\alpha}$. Then,

$$
\begin{aligned}
\mu(x) & \geq \alpha, \\
\mu(x * y) & \geq \alpha .
\end{aligned}
$$

This implies that $\alpha \leq \mu(x) \wedge \mu(x * y) \leq \mu(y)$ and hence $y \in \mu_{\alpha}$. So $\mu_{\alpha}$ is a deductive system of $A$.

Conversely, suppose that $\mu_{\alpha}$ is a deductive system of $A$ for all $\alpha \in L$. In particular, $\mu_{1}$ is a deductive system. Since $1 \in \mu_{1}$, we have $\mu(1) \geq 1$, but $\mu(x) \leq 1, \forall x \in A$, and hence $\mu(1) \leq 1$. Thus, we have $\mu(1)=1$. Again, let $x, y \in A$ and put $\alpha=\mu(x) \wedge \mu(x * y)$. This implies that $x \in \mu_{\alpha}$ and $x * y \in \mu_{\alpha}$ so we have $y \in \mu_{\alpha}$. Therefore,

$$
\mu(y) \geq \alpha=\mu(x) \wedge \mu(x * y) .
$$

Hence, $\mu$ is an $L$-fuzzy deductive system of $A$.

Definition 4. For any subset $S$ of $A$ and each $\alpha \in L-\{1\}$, define an $L$-fuzzy subset $\alpha_{S}$ of $A$ by for any $x \in A$,

$$
\alpha_{S}(x)= \begin{cases}1 & \text { if } x \in S, \\ \alpha & \text { otherwise. }\end{cases}
$$

Corollary 1. Let $S$ be a subset of $A$ and $1 \neq \alpha \in L$. Then, $S$ is a deductive system if and only if $\alpha_{S}$ is an L-fuzzy deductive system of $A$.

Proof. Suppose that $S$ is a deductive system of $A$ and let $\alpha \in L-\{1\}$. Then, $1 \in S$ and hence, $\alpha_{S}(1)=1$. Let $x, y \in A$. If either $\alpha_{S}(x)=\alpha$ or $\alpha_{S}(x * y)=\alpha$, then

$$
\alpha_{S}(x) \wedge \alpha_{S}(x * y)=\alpha \leq \alpha_{S}(y) .
$$

Assume that $\alpha_{S}(x) \neq \alpha$ and $\alpha_{S}(x * y) \neq \alpha . \quad$ Then, $x, x * y \in S$. By our assumption, $S$ is a deductive system of $A$ so that $y \in S$ and hence,

$$
\alpha_{S}(y)=1 \geq \alpha_{S}(x) \wedge \alpha_{S}(x * y) .
$$

Thus, $\alpha_{S}$ is an $L$-fuzzy deductive system of $A$. Conversely, suppose that $\alpha_{S}$ is an $L$-fuzzy deductive system of $A$ for some $\alpha \in L-\{1\}$. Then, $\alpha_{S}(1)=1$ and hence, $1 \in S$. Also, let $x, y \in A$ such that $x, x * y \in S$. Then, $\alpha_{S}(x)=1=\alpha_{S}(x * y)$, which gives $\alpha_{S}(x) \wedge \alpha_{S}(x * y)=1 . \alpha_{S}$ being an $L$-fuzzy deductive system, we obtain $\alpha_{S}(y)=1$. Equivalently, $y \in S$ and hence $S$ is a deductive system of $A$. This completes the proof.

Remark 1 . The $L$-fuzzy deductive system $\alpha_{S}$ given above is called the $\alpha$-level $L$-fuzzy deductive system of $A$ corresponding to the deductive system $S$.

Let us now define a binary operation $\odot$, called a product, on $L^{A}$ as follows: for any $\eta, \sigma \in L^{A}, \quad(\eta \odot \sigma)(x)=\mathrm{V}$ $\{\eta(a) \wedge \sigma(b): x=a * b\}$ for all $x \in A$.

In the following theorem, we use this product and give a Rosenfeld-type characterization for $L$-fuzzy subsets of $A$ to be an $L$-fuzzy deductive system.

Theorem 1. $\mu \in L^{A}$ is an $L$-fuzzy deductive system of $A$ if and only if $\mu(1)=1$ and for any normalized $\eta \in L^{A}$ and any $\sigma \in L^{A}, \eta \subseteq \mu$ and $\eta \odot \sigma \subseteq \mu$ together imply $\sigma \subseteq \mu$.

Proof. Suppose that $\mu$ is an $L$-fuzzy deductive system of $A$. Let $\eta$ be a normalized $L$-fuzzy subset of $A$ and $\sigma$ is an $L$-fuzzy subset of $A$ such that $\eta \subseteq \mu$ and $\eta \odot \sigma \subseteq \mu$. Then, $\eta(a)=1$ for some $a \in A$. Now, for any $x \in A$,

$$
\begin{aligned}
\sigma(x) & =1 \wedge \sigma(x) \\
& =\eta(a) \wedge \sigma(x) \\
& \leq(\eta \odot \sigma)(a * x) \\
& \leq \mu(a * x) \\
& =\eta(a) \wedge \mu(a * x) \quad(\text { since } \mu(y) \leq 1=\eta(a), \forall y \in A) \\
& \leq \mu(a) \wedge \mu(a * x) \leq \mu(x) .
\end{aligned}
$$

Therefore, $\sigma \subseteq \mu$.

Conversely, suppose that $\mu \in L^{A}$ satisfies the given condition. Now, we show that $\mu$ is an $L$-fuzzy deductive system of $A$. By hypothesis, $\mu(1)=1$. Let $x, y \in A$. Define $L$-fuzzy subsets $\eta$ and $\sigma$ of $A$ by for any $z \in A$,

$$
\begin{aligned}
& \eta(z)= \begin{cases}1, & \text { if } z=1, \\
\mu(x), & \text { if } z=x, \\
0, & \text { otherwise, }\end{cases} \\
& \sigma(z)= \begin{cases}1, & \text { if } z=1, \\
\mu(x * y), & \text { if } z=y, \\
0, & \text { otherwise. }\end{cases}
\end{aligned}
$$


Clearly $\eta$ is a normalized and $\eta \subseteq \mu$. Moreover, for each $z \in A$,

$$
(\eta \odot \sigma)(z)= \begin{cases}1, & \text { if } z=1, \\ \mu(x) \wedge \mu(x * y), & \text { if } z=x * y, \\ 0, & \text { otherwise }\end{cases}
$$

which is a subset of $\mu$. Hence, by hypothesis, we have $\sigma \subseteq \mu$. Hence, $\mu(x * y)=\sigma(y) \leq \mu(y)$ and so $\mu(x) \wedge \mu(x * y) \leq$ $\mu(y)$. Therefore, $\mu$ is an $L$-fuzzy deductive system.

Notation 1. We write $F \subset_{f} A$, to say that $F$ is a finite subset of $A$. The following result is another characterization for $L$-fuzzy deductive systems of $A$.

Theorem 2. An L-fuzzy subset $\mu$ of $A$ is an L-fuzzy deductive system of $A$ if and only if for any $F \subset_{f} A, \mu(x) \geq \wedge_{a \in F} \mu(a)$ for all $x \in D(F)$.

Proof. Suppose that $\mu$ is an $L$-fuzzy deductive system of $A$. Let $F \subset_{f} A$ and put $\alpha=\inf \{\mu(a): a \in F\}$. Then, $\mu(a) \geq \alpha$ for all $a \in F$ and hence $F \subseteq \mu_{\alpha}$. Clearly, by Lemma $3, \mu_{\alpha}$ is a deductive system. Therefore, $D(F) \subseteq \mu_{\alpha}$ and hence, $\mu(x) \geq \alpha=\wedge_{a \in F} \mu(a)$ for all $x \in D(F)$.

Conversely, suppose that $\mu$ satisfies the given conditions. Now, since $D(\varnothing)=\{1\}$, by hypothesis, we have $\mu(1) \geq \inf \{\mu(a): a \in \varnothing\}=1$ and hence $\mu(1)=1$.

Let $x, y \in A$. Put $F=\{x, x * y\}$. Then, it is easy to see that $y \in D(F)$. Thus, by hypothesis, we have $\mu(y) \geq \mu(x) \wedge \mu(x * y)$. Therefore, $\mu$ is an $L$-fuzzy deductive system of $A$.

\section{L-Fuzzy Deductive Systems Generated by L-Fuzzy Subset}

Lemma 4. The class $F D_{L}(A)$ is closed under arbitrary intersection.

Proof. Let $\left\{\mu_{i}: i \in \Delta\right\}$ be any family of $L$-fuzzy deductive systems of $A$. Now, we claim that $\cap_{i \in \Delta} \mu_{i}$ is an $L$-fuzzy deductive system of $A$. If $\Delta=\varnothing$, then by logical convention, $\cap_{i \in \Delta} \mu_{i}=\overline{1}$, where $\overline{1}$ is an $L$-fuzzy subset of $A$ defined by

$$
\overline{1}(x)=1,
$$

for all $x \in A$, and clearly, it is an $L$-fuzzy deductive system of $A$. Assume that $\Delta \neq \varnothing$. Since $\mu_{i}(1)=1$, for all $i \in \Delta$, we have $\cap_{i \in \Delta} \mu_{i}(1)=\inf \left\{\mu_{i}(1): i \in \Delta\right\}=1$. Again, let $x, y \in A$. Then,

$$
\begin{aligned}
\left(\bigcap_{i \in \Delta} \mu_{i}\right)(x) \wedge\left(\bigcap_{i \in \Delta} \mu_{i}\right)(x * y)= & \inf \left\{\mu_{i}(x): i \in \Delta\right\} \\
& \wedge \inf \left\{\mu_{i}(x * y): i \in \Delta\right\} \\
& =\inf \left\{\mu_{i}(x) \wedge \mu_{i}(x * y): i \in \Delta\right\} \\
\leq & \inf \left\{\mu_{i}(y): i \in \Delta\right\} \\
& =\left(\bigcap_{i \in \Delta} \mu_{i}\right)(y) .
\end{aligned}
$$

Therefore, $\cap_{i \in \Delta} \mu_{i}$ is an $L$-fuzzy deductive systems of $A$.

This lemma confirms that the class $\mathrm{FD}_{L}(A)$ of all $L$-fuzzy deductive systems of $A$ forms a closure fuzzy set system and for any $L$-fuzzy subset $\mu$ of $A$, always there exists a smallest $L$-fuzzy deductive system of $A$ containing $\mu$ which we call it the $L$-fuzzy deductive system of $A$ generated by $\mu$ and is denoted by $D_{L}(\mu)$.

Lemma 5. Let $\mu$ and $\eta$ be L-fuzzy subsets of $A$. Then,

(1) $\mu \in F D_{L}(A)$, if and only if $D_{L}(\mu)=\mu$,

(2) $\mu \subseteq \eta \Rightarrow D_{L}(\mu) \subseteq D_{L}(\eta)$,

(3) $D_{L}\left(D_{L}(\mu)\right)=D_{L}(\mu)$.

It can be deduced from this lemma that the map $\mu \longmapsto D_{L}(\mu)$ forms a closure operator, i.e., isotone, extensive and idempotent on the lattice $L^{A}$. Moreover, $L$-fuzzy deductive systems of $A$ are those closed elements of $L^{A}$ with respect to this closure operator.

Lemma 6. For any subset $S$ of $A$ and each $\alpha \in L-\{1\}$, $\alpha_{D(S)}=D_{L}\left(\alpha_{S}\right)$.

Proof. We show that $\alpha_{D(S)}$ is the smallest $L$-fuzzy deductive system of $A$ containing $\alpha_{S}$. Since $D(S)$ is a deductive system of $A$ containing $S$, it is clear that $\alpha_{D(S)}$ is an $L$-fuzzy deductive system of $A$ containing $\alpha_{S}$. Suppose that $\lambda$ is an $L$-fuzzy deductive system of $A$ such that $\alpha_{S} \subseteq \lambda$. Then, $\lambda(s)=1$ for all $s \in S$. Let $z$ be any element in $A$. If $z \notin D(S)$, then $\alpha_{D(S)}(z)=\alpha \leq \lambda(z)$. Let $z \in D(S)$. Since $\alpha_{S} \subseteq \lambda$, we have $S \subseteq \lambda_{1}$. This implies that $D(S) \subseteq D\left(\lambda_{1}\right)=\lambda_{1}$. So $z \in \lambda_{1}$ and hence $\lambda(z)=1$. Therefore, $\alpha_{D(S)}(z)=1=\lambda(z)$ so that $\alpha_{D(S)} \subseteq \lambda$. Therefore, $\alpha_{D(S)}=D_{L}\left(\alpha_{S}\right)$.

Corollary 2. Let $S$ be any subset of $A$ and $\chi_{S}$ its characteristic function. Then, $D_{L}\left(\chi_{S}\right)=\chi_{D(S)}$.

In the following theorem, we characterize $L$-fuzzy deductive systems generated by an $L$-fuzzy subset in terms of their level sets.

Theorem 3. For an L-fuzzy subset $\lambda$ of $A$, let $\hat{\lambda}$ be an $L$-fuzzy subset of $A$ defined by

$$
\widehat{\lambda}(x)=\vee\left\{\alpha \in L: x \in D\left(\lambda_{\alpha}\right)\right\},
$$

for all $x \in A$. Then, $\hat{\lambda}=D_{L}(\lambda)$.

Proof. We show that $\hat{\lambda}$ is the smallest $L$-fuzzy deductive system of $A$ containing $\lambda$. Let us first show that $\hat{\lambda}$ is an $L$-fuzzy deductive system:

(1) $\hat{\lambda}(1)=\vee\left\{\alpha \in L: 1 \in D\left(\lambda_{\alpha}\right)\right\}=1$.

(1) Let $x, y \in A$. Then, consider

$$
\begin{aligned}
\hat{\lambda}(x) \wedge \hat{\lambda}(x * y) & =\vee\left\{\alpha \in L: x \in D\left(\lambda_{\alpha}\right)\right\} \wedge \vee\left\{\beta \in L: x * y \in D\left(\lambda_{\beta}\right)\right\} \\
& =\vee\left\{\alpha \wedge \beta: x \in D\left(\lambda_{\alpha}\right), x * y \in D\left(\lambda_{\beta}\right)\right\} .
\end{aligned}
$$


If we put $\gamma=\alpha \wedge \beta$, then we obtain $\lambda_{\alpha} \subseteq \lambda_{\gamma}$ and $\lambda_{\beta} \subseteq \lambda_{\gamma}$, which gives the following:

$$
\begin{aligned}
& D\left(\lambda_{\alpha}\right) \subseteq D\left(\lambda_{\gamma}\right), \\
& D\left(\lambda_{\beta}\right) \subseteq D\left(\lambda_{\gamma}\right) .
\end{aligned}
$$

Therefore, $x, x * y \in D\left(\lambda_{\gamma}\right)$ and hence $y \in D\left(\lambda_{\gamma}\right)$. Now it follows from the above-given equality that

$$
\begin{aligned}
\widehat{\lambda}(x) \wedge \widehat{\lambda}(x * y) & =\vee\left\{\alpha \wedge \beta: x \in D\left(\lambda_{\alpha}\right), x * y \in D\left(\lambda_{\beta}\right)\right\} \\
& \leq \vee\left\{\gamma \in L: y \in D\left(\lambda_{\gamma}\right)\right\} \\
& =\widehat{\lambda}(y) .
\end{aligned}
$$

Therefore, $\hat{\lambda}$ is an $L$-fuzzy deductive system of $A$. It is also clear to see that $\lambda \subseteq \widehat{\lambda}$. Suppose that $\mu$ is any other $L$-fuzzy deductive system of $A$ such that $\lambda \subseteq \mu$. Then, it is clear that $D\left(\lambda_{\alpha}\right) \subseteq \mu_{\alpha}$ for all $\alpha \in L$. Now, for any $x \in A$, consider

$$
\widehat{\lambda}(x)=\vee\left\{\alpha \in L: x \in D\left(\lambda_{\alpha}\right)\right\} \leq \vee\left\{\alpha \in L: x \in \mu_{\alpha}\right\}=\mu(x) \text {. }
$$

Therefore, $\hat{\lambda}$ is the smallest $L$-fuzzy deductive system containing $\lambda$ and hence $\hat{\lambda}=D_{L}(\lambda)$.

In the following theorem, we characterize $L$-fuzzy deductive systems generated by an $L$-fuzzy subset using finitely generated crisp deductive systems.

Theorem 4. For an L-fuzzy subset $\lambda$ of $A$, let $\bar{\lambda}$ be an L-fuzzy subset of $A$ defined by

$$
\bar{\lambda}(x)=\vee\left\{\wedge_{a \in F} \lambda(a): x \in D(F), F \subset{ }_{f} A\right\},
$$

for all $x \in A$. Then, $\bar{\lambda}=D_{L}(\lambda)$.

Proof. By Theorem 3, it is enough if we show that $\bar{\lambda}=\widehat{\lambda}$. For each $x \in A$, let us define two sets $H_{x}$ and $G_{x}$ as follows:

$$
\begin{aligned}
H_{x} & =\left\{\wedge_{a \in F} \lambda(a): x \in D(F), F \subset_{f} A\right\}, \\
G_{x} & =\left\{\alpha \in L: x \in D\left(\lambda_{\alpha}\right)\right\} .
\end{aligned}
$$

Our claim is to show that

$$
\vee\left\{\alpha: \alpha \in H_{x}\right\}=\vee\left\{\alpha: \alpha \in G_{x}\right\} .
$$

In one way, we show that $H_{x} \subseteq G_{x}$. If $\alpha \in H_{x}$, then $\alpha=$ $\wedge_{a \in F} \lambda(a)$ and $x \in D(F)$ for some finite subset $F$ of $A$. That is, $a \in \lambda_{\alpha}$, for all $a \in F$ and $x \in D(F)$. Hence, $x \in D\left(\lambda_{\alpha}\right)$. Then, $\alpha \in G_{x}$ and hence $H_{x} \subseteq G_{x}$. In the other way, we prove that, for each $\alpha \in G_{x}$, there exists $\beta \in H_{x}$ such that $\alpha \leq \beta$. Let $\alpha \in G_{x}$. Then, $x \in D\left(\lambda_{\alpha}\right)$; that is, there exist $x_{1}, x_{2}, \ldots, x_{n} \in \lambda_{\alpha}$ such that $x_{1} *\left(x_{2} *\left(\cdots\left(x_{n} *\right.\right.\right.$ $x) \cdots))=1$. This implies that $\wedge_{i=1}^{n} \lambda\left(a_{i}\right) \geq \alpha$.

Moreover, if we put $\beta=\wedge_{i=1}^{n} \lambda\left(x_{i}\right)$ and $F=\left\{x_{1}, x_{2}, \ldots, x_{n}\right\}$, then $F$ is a finite subset of $A$ such that $x \in D(F)$. Thus, $\beta \in H_{x}$ such that $\alpha \leq \beta$.
Corollary 3. For each $x \in A$ and $\alpha \in L-\{0\}$, the L-fuzzy deductive system of A generated by the fuzzy point $x_{\alpha}$ is characterized as

$$
D_{L}\left(x_{\alpha}\right)(z)= \begin{cases}1, & \text { if } z=1, \\ \alpha, & \text { if } z \in D(\{\mathrm{x}\})-\{1\}, \\ 0, & \text { otherwise, }\end{cases}
$$

for all $z \in A$.

The following theorem gives a description for $\alpha$-level cuts of $L$-fuzzy deductive systems generated by an $L$-fuzzy subset.

Theorem 5. Let $\mu$ be an L-fuzzy subset of $A$ and $\alpha \in L$ :

$$
\left(D_{L}(\mu)\right)_{\alpha}=\bigcup\left\{\underset{\gamma \in M}{\cap} D\left(\mu_{\gamma}\right): M \subseteq L \text { and } \alpha \leq \sup M\right\} .
$$

Proof. Let us put

$$
H=\bigcup\left\{\bigcap_{\gamma \in M} D\left(\mu_{\gamma}\right): M \subseteq L \text { and } \alpha \leq \sup M\right\} .
$$

If $x \in H$, then $x \in \cap_{\gamma \in M} D\left(\mu_{\gamma}\right)$ for some $M \subseteq L$ with $\alpha \leq \sup M$; i.e., $x \in D\left(\mu_{\gamma}\right)$ for all $\gamma \in M$ and $\alpha \leq \sup M$. By Theorem 3, we have the following:

$$
D_{L}(\mu)(x)=\vee\left\{\beta \in L: x \in D\left(\mu_{\beta}\right)\right\} .
$$

Therefore, $D_{L}(\mu)(x) \geq \gamma$ for all $\gamma \in M$. This gives $D_{L}(\mu)(x) \geq \alpha$. Thus, $x \in\left(D_{L}(\mu)\right)_{\alpha}$ and hence $H \subseteq\left(D_{L}(\mu)\right)_{\alpha}$. To prove the other inequality, let us take $x \in\left(D_{L}(\mu)\right)_{\alpha}$. Then,

$$
\vee\left\{\beta \in L: x \in D\left(\mu_{\beta}\right)\right\} \geq \alpha .
$$

If we put $M=\left\{\beta \in L: x \in D\left(\mu_{\beta}\right)\right\}$, then $M \subseteq L$ such that $\alpha \leq \sup M$ and $x \in D\left(\mu_{\gamma}\right)$ for all $\gamma \in M$. This means that $x \in \cap_{\gamma \in M} D\left(\mu_{\gamma}\right)$ and $\alpha \leq \sup M$. Thus, $x \in H$, and hence, the proof ends.

Corollary 4. For any L-fuzzy subset $\mu$ of $A$ and each $\alpha \in L$, $D\left(\mu_{\alpha}\right) \subseteq\left(D_{L}(\mu)\right)_{\alpha}$. Moreover, if $L$ is a chain and $\mu$ is finite valued or equivalently if $\mu$ has sup property, then the equality holds.

In the following, we give an algebraic characterization for $L$-fuzzy deductive systems generated by $L$-fuzzy subset. Let $\mu$ be an $L$-fuzzy subset of $A$ and $\mathbb{N}$ be a set of positive integers. For each $n \in \mathbb{N}$, let us define $L$-fuzzy subsets $\mu_{n}$ of $A$ as follows:

$$
\mu_{1}(x)= \begin{cases}1, & \text { if } x=1, \\ \mu(x), & \text { otherwise, }\end{cases}
$$

for all $x \in A$, and for each $n \geq 1$ and each $y \in A$,

$$
\mu_{n+1}(y)=\vee\left\{\mu_{n}(x) \wedge \mu_{n}(x * y): x \in A\right\} .
$$

Then, it is immediate from the definition that $\mu_{n}(1)=1$ for all $n \geq 1$. Moreover, one can deduce that

$$
\mu_{1} \subseteq \mu_{2} \subseteq \mu_{3} \subseteq \cdots \subseteq \mu_{n} \subseteq \cdots
$$


Theorem 6. Let $\mu$ be an L-fuzzy subset of $A$. Then, $D_{L}(\mu)=\bigcup\left\{\mu_{n}: n \in \mathbf{N}\right\}$.

Proof. Put $\bar{\mu}=\bigcup\left\{\mu_{n}: n \in N\right\}$. Now, we claim that $\bar{\mu}$ is the smallest $L$-fuzzy deductive system containing $\mu$. Now, let $x \in A$. Then,

$$
\begin{aligned}
\bar{\mu}(x) & =\vee\left\{\mu_{n}(x): n \in \mathbb{N}\right\} \\
& \geq \mu_{1}(x) \\
& \geq \mu(x) .
\end{aligned}
$$

Thus, we have $\mu \subseteq \bar{\mu}$.

Since $\bar{\mu}(1) \geq \mu_{1}(1)=1$, we have $\bar{\mu}(1)=1$. Let $x, y \in A$ and $x \neq 1$.

Then

$$
\begin{aligned}
\bar{\mu}(x) \wedge \bar{\mu}(x * y)= & \vee\left\{\mu_{n}(x): n \in \mathbb{N}\right\} \wedge \vee\left\{\mu_{m}(x * y): m \in \mathbb{N}\right\} \\
= & \vee\left\{\mu_{n}(x) \wedge \mu_{m}(x * y): n, m \in \mathbb{N}\right\} \\
\leq & \vee\left\{\mu_{k}(x) \wedge \mu_{k}(x * y):\right. \text { where } \\
& k=\max \{m, n\}, n, m \in \mathbb{N}\} \\
= & \mu_{k+1}(y) \\
\leq & \vee\left\{\mu_{n}(y): n \in \mathbb{N}\right\} \\
= & \bar{\mu}(y) .
\end{aligned}
$$

So $\bar{\mu}$ is an $L$-fuzzy deductive system of $A$. Again, let $\theta$ be any $L$-fuzzy deductive system of $A$ such that $\mu \subseteq \theta$. We show that $\mu_{n} \subseteq \theta$ for all $n \in \mathbb{N}$. We use induction on $n$. Let $x \in A$. Then, if $x=1$, then $\theta(1)=1=\mu_{1}(x)$. If $x \neq 1$, then $\theta(x) \geq \mu(x)=\mu_{1}(x)$. This implies that $\mu_{1} \subseteq \theta$ and hence it holds for $n=1$. Let $n>1$ and assume the result to be true for some $n-1$, i.e., $\mu_{n-1} \subseteq \theta$. Now, for any $x \in A$, we have

$$
\begin{aligned}
\mu_{n}(x) & =\vee\left\{\mu_{n-1}(a) \wedge \mu_{n-1}(a * x)\right. \\
& \leq \vee\{\theta(a) \wedge \theta(a * x) \leq \theta(x) .
\end{aligned}
$$

Hence, $\mu_{n} \subseteq \theta$. Thus, by mathematical induction, we have $\mu_{n} \subseteq \theta$ for all $n \in \mathbb{N}$. Hence, $\bar{\mu}=\bigcup\left\{\mu_{n}: n \in N\right\} \subseteq \theta$. Therefore, $\bar{\mu}=D_{L}(\mu)$.

The following is also another algebraic characterization of $L$-fuzzy deductive system generated by an $L$-fuzzy subset of $A$.

Theorem 7. Let $\lambda$ be an L-fuzzy subset of $A$. Then, the L-fuzzy subset $\bar{\lambda}$ defined by: $\bar{\lambda}(1)=1$ and for $x \neq 1$,

$$
\begin{aligned}
\bar{\lambda}(x)= & \vee\left\{\wedge_{i=1}^{n} \lambda\left(x_{i}\right): x_{1} *\left(x_{2} *\left(\cdots\left(x_{n} * x\right) \cdots\right)\right)=1,\right. \\
& \left.x_{1}, \ldots, x_{n} \in A\right\},
\end{aligned}
$$

is the L-fuzzy deductive system of A generated by $\lambda$.

The following theorem gives a method of constructing $L$-fuzzy deductive systems using crisp deductive systems in the sense of [10].

Theorem 8. Suppose that $\left\{H_{\alpha}\right\}_{\alpha \in L}$ is a family of deductive systems of $A$ such that

$$
\bigcap_{\alpha \in M} H_{\alpha}=H_{\sup M}
$$

for all $M \subseteq L$. Then, there is a unique L-fuzzy deductive system $\mu$ of $A$ for which $\mu_{\alpha}=H_{\alpha}$ for all $\alpha \in L$. Moreover, every $L$-fuzzy deductive system of $A$ is obtained in this way only.

Proof. We first show that the map $\alpha \longmapsto H_{\alpha}$ is antitone, in the sense that, for each $\alpha, \beta \in L, \alpha \leq \beta \Longrightarrow H_{\beta} \subseteq H_{\alpha}$. Let $\alpha, \beta \in L$ such that $\alpha \leq \beta$. Put $M=\{\alpha, \beta\}$. Then, supM $=\beta$. By our hypothesis $H_{\alpha} \cap H_{\beta}=H_{\sup M}=H_{\beta}$. Therefore, $H_{\beta} \subseteq H_{\alpha}$ and hence, the map $\alpha \longmapsto H_{\alpha}$ is antitone. Define an $L$-fuzzy subset $\mu$ of $A$ by

$$
\mu(x)=\vee\left\{\alpha \in L: x \in H_{\alpha}\right\},
$$

for all $x \in A$. Clearly, $\mu$ is well defined. Our aim is to show that $\mu_{\alpha}=H_{\alpha}$ for all $\alpha \in L$. The inclusion $H_{\alpha} \subseteq \mu_{\alpha}$ follows easily from the definition of $\mu$. To prove the other inclusion, let $x \in \mu_{\alpha}$. Then, $\mu(x) \geq \alpha$, i.e.,

$$
\vee\left\{\gamma \in L: x \in H_{\gamma}\right\} \geq \alpha .
$$

If we put $M=\left\{\gamma \in L: x \in H_{\gamma}\right\}$, then $M \subseteq L$ such that $\alpha \leq \operatorname{supM}$ and $x \in H_{\gamma}$ for all $\gamma \in M$, i.e.,

$$
x \in \bigcap_{\gamma \in M} H_{\gamma} \text {. }
$$

By our assumption, it follows that $x \in H_{\text {sup } M}$. Since $\alpha \leq \sup M$ and the map $\alpha \longmapsto H_{\alpha}$ is antitone, we obtain $x \in H_{\alpha}$. Thus, $H_{\alpha}=\mu_{\alpha}$. This means that $\mu$ is an $L$-fuzzy subset of $A$ for which its $\alpha$-level sets are $H_{\alpha}$ 's. Each $H_{\alpha}$ being a deductive system of $A$, it follows from Lemma 3 that $\mu$ is an $L$-fuzzy deductive system. The uniqueness of $\mu$ follows from the fact $\mu_{\alpha}=H_{\alpha}$ for all $\alpha \in L$. The converse is straightforward.

\section{Lattice of $\boldsymbol{L}$-Fuzzy Deductive Systems}

Let us first recall some important results from [24]. Let $\mathscr{C}$ be a nonempty collection of fuzzy subsets of a nonempty set $X$.

Definition 5. $\mathscr{C}$ is said to be a closure system in $L^{X}$ if it is closed under arbitrary intersection of fuzzy sets; i.e., if for any subcollection $\mathscr{D}$ of fuzzy subsets of $X$ in $\mathscr{C}$, it holds that

$$
\bigcap_{\mu \in \mathscr{D}} \mu \in \mathscr{C} \text {. }
$$

A closure system of fuzzy sets is also known as the "Moor family" of fuzzy sets.

Remark 2. If $\mathscr{C}$ is a closure system in $L^{X}$, then it contains the fuzzy subset $1_{X}$ of $X$. This is because the fuzzy set $1_{X}$ can be expressed as the infimum of an empty collection of fuzzy subsets of $X$.

Theorem 9. If $\mathscr{C}$ is a closure system in $L^{X}$, then $(\mathscr{C}, \subseteq)$ forms a complete lattice, where $\subseteq$ is the inclusion ordering of fuzzy sets. 
Definition 6. A nonempty collection $\mathscr{C}$ of fuzzy subsets of $X$ is called inductive if every nonempty chain in $\mathscr{C}$ has a supremum in $\mathscr{C}$.

Definition 7. A closure system $\mathscr{C}$ in $L^{X}$ is said to be an algebraic closure fuzzy sets system if it is inductive.

As observed in the previous section, the collection $F D_{L}(A)$ of all $L$-fuzzy deductive systems of $A$ is closed under arbitrary intersection of fuzzy sets. Thus, $\left(F D_{L}(A), \subseteq\right)$ forms a closure fuzzy set system and hence it is a complete lattice. The following theorem summarizes this.

Theorem 10. The collection $F D_{L}(A)$ forms a complete lattice with respect to the pointwise ordering, where the infimum and supremum of any of its subfamily $\left\{\mu_{i}: i \in \Delta\right\}$, respectively, are

$$
\begin{aligned}
\inf _{i \in \Delta} \mu_{i} & =\bigcap_{i \in \Delta} \mu_{i}, \\
\sup _{i \in \Delta} \mu_{i} & =D_{L}\left(\cup_{i \in \Delta} \mu_{i}\right) .
\end{aligned}
$$

Corollary 5. The set $F D_{L}(A)$ forms the algebraic closure fuzzy set system on $A$ with respect to the pointwise ordering of fuzzy sets.

Proof 13. It is enough to show that $F D_{L}(A)$ is inductive in $L^{A}$. Let $\left\{\mu_{i}\right\}_{i \in \Delta}$ be a chain in $F D_{L}(A)$. Let us put

$$
\eta=\cup_{i \in \Delta} \mu_{i} .
$$

We show that $\eta$ is an $L$-fuzzy deductive system of $A$. Clearly, $\eta(1)=1$. Let $x, y \in A$. First observe that, for each $i, j \in \Delta$, there exists $k \in\{i, j\}$ such that $\mu_{i} \subseteq \mu_{K}$ and $\mu_{j} \subseteq \mu_{k}$. Now, consider the following:

$$
\begin{aligned}
\eta(x) \wedge \eta(x * y) & =\left(\vee_{i \in \Delta} \mu_{i}(x)\right) \wedge\left(\vee_{j \in \Delta} \mu_{j}(x * y)\right) \\
& =\bigvee_{i, j \in \Delta} \mu_{i}(x) \wedge \mu_{j}(x * y) \\
& \leq \vee_{k \in \Delta}\left(\mu_{k}(x) \wedge \mu_{k}(x * y)\right) \\
& \leq \underset{k \in \Delta}{\vee} \mu_{k}(y) \\
& =\eta(y) .
\end{aligned}
$$

Therefore $\eta$ is an $L$-fuzzy deductive system of $A$.

Remark 3. If $L$ is an algebraic lattice, then $F C_{a}(A)$ forms an algebraic lattice.

\section{L-Fuzzy Congruences}

By an $L$-fuzzy relation on $A$, we mean an $L$-fuzzy subset of $A \times A$. For any $\alpha \in L$ and an $L$-fuzzy relation $\Theta$ on $A$, the set

$$
\Theta_{\alpha}=\{(x, y) \in A \times A: \Theta(x, y) \geq \alpha\},
$$

is called the $\alpha$-level relation of $\Theta$ on $A$.

Definition 8. An $L$-fuzzy relation $\Theta$ on $A$ is said to be
(1) Reflexive if $\Theta(x, x)=1$ for all $x \in A$

(2) Symmetric if $\Theta(x, y)=\Theta(y, x)$ for all $x, y \in A$

(3) Transitive if for each $x, z \in A, \quad \Theta(x, z) \geq \Theta$ $(x, y) \wedge \Theta(y, z)$ for all $y \in A$

A reflexive, symmetric, and transitive $L$-fuzzy relation on $A$ is called an $L$-fuzzy equivalence relation on $A$.

Definition 9. An $L$-fuzzy relation $\Theta$ on $A$ is said to be compatible, if

$$
\Theta(a * c, b * d) \geq \Theta(a, b) \wedge \Theta(c, d),
$$

for all $a, b, c, d \in A$.

This fact is often expressed in the way that $\Theta$ is said to have the substitution property with respect to the binary operation $*$.

Definition 10. A compatible $L$-fuzzy equivalence relation on $A$ is called an $L$-fuzzy congruence relation on $A$.

We denote by FCon $(A)$ the class of all $L$-fuzzy congruence relations on $A$, and it is clear that $F \operatorname{Con}(A)$ is a complete lattice. For any $a \in L$ and $\Theta \in F \operatorname{Con}(A)$, define a fuzzy subset $[a]_{\Theta}$ of $A$ by

$$
[a]_{\Theta}(x)=\Theta(a, x),
$$

for all $x \in A$. We call $[a]_{\Theta}$ an $L$-fuzzy congruence class of $\Theta$ determined by $a$, and in particular, $[1]_{\Theta}$ is called the kernel of $\Theta$. Let us put

$$
\frac{A}{\Theta}=\left\{[a]_{\Theta}: a \in A\right\}
$$

and define a binary operation $\circledast$ on $A / \Theta$ by

$$
[a]_{\Theta} \circledast[b]_{\Theta}=[a * b]_{\Theta} .
$$

Then, it is routine to verify that $\left((A / \Theta), \circledast,[1]_{\Theta}\right)$ is a Hilbert algebra and it is called the quotient Hilbert algebra of $A$ modulo $\Theta$.

Lemma 7. For each $\Theta \in F \operatorname{Con}(A),[1]_{\Theta}$ is an L-fuzzy deductive system of $A$.

Proof. Since $\Theta$ is reflexive, we have $[1]_{\Theta}(1)=\Theta(1,1)=1$. Let $x, y \in A$. Then,

$$
\begin{aligned}
{[1]_{\Theta}(x) \wedge[1]_{\Theta}(x * y) } & =\Theta(1, x) \wedge \Theta(1, x * y) \\
& =\Theta(1, x) \wedge \Theta(y, y) \wedge \Theta(1, x * y) \\
& \leq \Theta(y,(x * y)) \wedge \Theta(1, x * y) \\
& \leq \Theta(1 * y,(x * y) *(x * y)) \\
& \leq \Theta(y, 1)=[1]_{\Theta}(y) .
\end{aligned}
$$

Thus, $[1]_{\Theta}$ is an $L$-fuzzy deductive system of $A$.

Remark 4. It is sufficient for $\Theta$ to be reflexive and compatible only to obtain the result of Lemma 7. 
Lemma 8. For each $\Theta, \Phi \in F \operatorname{Con}(A)$,

$$
[1]_{\Theta}=[1]_{\Phi} \Longrightarrow \Theta=\Phi \text {. }
$$

Proof. Let $\Theta, \Phi \in \mathrm{FCon}(A)$ such that $[1]_{\Theta}=[1]_{\Phi}$. Then, it is clear that $\Theta_{\alpha}[1]=\Phi_{\alpha}[1]$ for all $\alpha \in L$. Let $(x, y) \in A \times A$, and put $\Theta(x, y)=\alpha$. Then, it is clear that $\Theta_{\alpha}$ is a congruence relation on $A$ containing $(x, y)$ and hence $(x * y, 1)$ and $(y * x, 1)$ belong to $\Theta_{\alpha}$. This imply that $x * y, y * x \in \Theta_{\alpha}[1]=\Phi_{\alpha}[1]$ and so the following hold in the quotient Hilbert algebra $\left(A / \Phi_{\alpha}\right)$ :

$$
\begin{aligned}
& \Phi_{\alpha}[x] \Phi_{\alpha}[y]=\Phi_{\alpha}[x * y]=\Phi_{\alpha}[1] \\
& \Phi_{\alpha}[y] \Phi_{\alpha}[x]=\Phi_{\alpha}[y * x]=\Phi_{\alpha}[1] .
\end{aligned}
$$

$\left(A / \Phi_{\alpha}\right)$ Since the binary operations $x * y$ and $y * x$ form a Gödel equivalence system in the variety of Hilbert algebras, it follows that $\Phi_{\alpha}[x]=\Phi_{\alpha}[y]$. Therefore, $(x, y) \in \Phi_{\alpha}$ and thus, $\Phi(x, y) \geq \alpha=\Theta(x, y)$. Similarly, we can show that $\Theta(x, y) \geq \Phi(x, y)$ so that the equality holds.

Corollary 6. For each $\Theta, \Phi \in \operatorname{FCon}(A)$,

$$
[1]_{\Theta} \subseteq[1]_{\Phi} \Longleftrightarrow \Theta \subseteq \Phi \text {. }
$$

Proof. Let $\Theta, \Phi \in \mathrm{FCon}(A)$ such that $[1]_{\Theta} \subseteq[1]_{\Phi}$. Then, it is clear that $\Theta_{\alpha}[1] \subseteq \Phi_{\alpha}[1]$ for all $\alpha \in L$. Let $x, y \in A$, and put $\Theta(x, y)=\alpha$. Then, it is clear that $\Theta_{\alpha}$ is a congruence relation on $A$ containing $(x, y)$ and hence $(x * y, 1)$ and $(y * x, 1)$ belong to $\Theta_{\alpha}$. This imply that $x * y, y * x \in \Theta_{\alpha}[1] \subseteq \Phi_{\alpha}[1]$ and so the following hold in the quotient Hilbert algebra $\left(A / \Phi_{\alpha}\right)$ :

$$
\begin{aligned}
& \Phi_{\alpha}[x] \Phi_{\alpha}[y]=\Phi_{\alpha}[x * y]=\Phi_{\alpha}[1] \\
& \Phi_{\alpha}[y] \Phi_{\alpha}[x]=\Phi_{\alpha}[y * x]=\Phi_{\alpha}[1]
\end{aligned}
$$

$\left(A / \Phi_{\alpha}\right)$ Since the binary operations $x * y$ and $y * x$ form a Gödel equivalence system in the variety of Hilbert algebras, it follows that $\Phi_{\alpha}[x]=\Phi_{\alpha}[y]$. Therefore, $(x, y) \in \Phi_{\alpha}$ and hence $\Phi(x, y) \geq \alpha=\Theta(x, y)$. Thus $\Theta \subseteq \Phi$.

Corollary 7. For each $\Theta, \Phi \in \operatorname{FCon}(A)$,

(1) $[1]_{\Theta \cap \Phi}=[1]_{\Theta} \cap[1]_{\Phi}$,

(2) $[1]_{\Theta \vee \Phi}=[1]_{\Theta} \vee[1]_{\Phi}$.

For any $L$-fuzzy subset $\mu$ of $A$, let us define an $L$-fuzzy relation $\Theta_{\mu}$ of $A$ as follows:

$$
\Theta_{\mu}(a, b)=\mu(a * b) \wedge \mu(b * a),
$$

for all $a, b \in A$.
Lemma 9. If the fuzzy relation $\Theta_{\mu}$ is reflexive, then the kernel of $\Theta_{\mu}$ is $\mu$.

Proof. Let $\Theta_{\mu}$ be reflexive. Then, for any $x \in A$, $1=\Theta_{\mu}(x, x)=\mu(x * x) \wedge \mu(x * x)=\mu(1)$. Thus, $\mu(1)=1$. Now, since for any $x \in A$,

$$
\begin{aligned}
{[1]_{\Theta_{\mu}}(x) } & =\Theta_{\mu}(1, x) \\
& =\mu(1 * x) \wedge \mu(x * 1)=\mu(x) \wedge \mu(1) \\
& =\mu(x) \wedge 1=\mu(x) .
\end{aligned}
$$

Therefore, the kernel of $\Theta_{\mu}$ is $\mu$.

Lemma 10. If $\Theta_{\mu}$ be an L-fuzzy equivalence relation on $A$, then for any $a, b \in A$ with $a \leq b$, it holds that

$$
\mu(b) \geq \mu(a) \wedge \mu(b * a) .
$$

Proof. Let $\Theta_{\mu}$ be an $L$-fuzzy equivalence relation on $A$ and $a, b \in A$ such that $a \leq b$. Then, $a * b=1$. By Lemma 9, we have $\mu(1)=1=\mu(a * b)$ and $[1]_{\Theta_{\mu}}=\mu$. Thus,

$$
\begin{aligned}
\mu(a) \wedge \mu(b * a) & =\mu(a) \wedge \mu(b * a) \wedge \mu(a * b) \\
& =\mu(a) \wedge \Theta_{\mu}(a, b) \\
& =[1]_{\Theta_{\mu}}(a) \wedge \Theta_{\mu}(a, b) \\
& =\Theta_{\mu}(1, a) \wedge \Theta_{\mu}(a, b) \\
& \leq \Theta_{\mu}(1, b)=[1]_{\Theta_{\mu}}(b)=\mu(b) .
\end{aligned}
$$

This proves the result.

Theorem 11. If $\mu$ is an L-fuzzy deductive system of $A$, then $\Theta_{\mu}$ is an L-fuzzy congruence relation on $A$ whose kernel is $\mu$.

Proof. Let $\mu$ be an $L$-fuzzy deductive system of $A$. Then, for any $x \in A, \Theta_{\mu}(x, x)=\mu(x * x) \wedge \mu(x * x)=\mu(1)=1$. Thus, $\Theta_{\mu}$ is reflexive. Again, for any $x, y \in A, \Theta_{\mu}(x, y)=$ $\mu(x * y) \wedge \mu(y * x)=\mu(y * x) \wedge \mu(x * y)=\Theta_{\mu}(y, x)$. Thus, $\Theta_{\mu}$ is symmetric. Let $x, y, z \in A$. Now,

$$
\begin{aligned}
\Theta_{\mu}(x, y) \wedge \Theta_{\mu}(y, z)= & (\mu(x * y) \wedge \mu(y * x)) \wedge(\mu(y * z) \\
& \wedge \mu(z * y)) \\
= & (\mu(x * y) \wedge \mu(y * z)) \wedge(\mu(z * y) \\
& \wedge \mu(y * x)) \\
\leq & \mu(x * z) \wedge \mu(z * x) \quad \text { (by, Lemma } 2) \\
= & \Theta_{\mu}(x, z) .
\end{aligned}
$$

Thus, $\Theta_{\mu}$ is transitive. Let $x, y, a, b \in A$. Now using Lemma 1 and Lemma 2, we have 


$$
\begin{aligned}
\Theta_{\mu}(x, y) \wedge \Theta_{\mu}(a, b)= & (\mu(x * y) \wedge \mu(y * x)) \wedge(\mu(a * b) \\
& \wedge \mu(b * a)) \\
= & (\mu(y * x) \wedge \mu(a * b)) \wedge(\mu(x * y) \\
& \wedge \mu(b * a)) \\
\leq & ((\mu(x * a) *(y * a)) \wedge \mu((y * a) \\
& *((y * b))) \wedge(\mu((y * b) *(x * b)) \\
& \wedge \mu((x * b) *(x * a))) \\
\leq & \mu((x * a) *(y * b)) \wedge \mu((y * b) \\
& *(x * a)) \\
= & \Theta_{\mu}(x * a, y * b) .
\end{aligned}
$$

Thus, $\Theta_{\mu}$ is compatible. Therefore, $\Theta_{\mu}$ is an $L$-fuzzy congruence relation on $A$ and its kernel, by Lemma 9, is $\mu$.

Corollary 8. The map $\Theta \mapsto[1]_{\Theta}$ is a lattice isomorphism between $\mathrm{FCon}(A)$ and $F D_{L}(A)$.

Proof. Lemma 8 confirms that the map is one-one. Moreover, for any $\mu \in F D_{L}(A)$, it is proved in the above theorem that $\Theta_{\mu} \in F \operatorname{Con}(A)$ such that $[1]_{\Theta_{\mu}}=\mu$. Then, the map $\Theta \longmapsto[1]_{\Theta}$ is onto. Furthermore, it is proved in Corollary 7 that this map is a lattice homomorphism. Therefore, it is a lattice isomorphism between $F C o n(A)$ and $F D_{L}(A)$.

Theorem 12. For a Hilbert Algebra, the lattice $\left(F D_{L}(A), \subseteq\right)$ is distributive with respect to the pointwise ordering ${ }^{\prime} \subseteq^{\prime}$.

\section{Data Availability}

No data were used to support the results of this study.

\section{Conflicts of Interest}

The authors declare that there are no conflicts of interest regarding the publication of this paper.

\section{Acknowledgments}

This research work was partially supported by a research grant from the College of Science, Bahir Dar University.

\section{References}

[1] L. A. Zadeh, "Fuzzy sets," Information and Control, vol. 8, no. 3, pp. 338-353, 1965.

[2] A. Rosenfeld, "Fuzzy groups," Journal of Mathematical Analysis and Applications, vol. 35, no. 3, pp. 512-517, 1971.

[3] N. Ajmal and K. V. Thomas, "Fuzzy lattices," Information Sciences, vol. 79, no. 3-4, pp. 271-291, 1994.

[4] B. A. Alaba and G. M. Addis, " $L$-fuzzy ideals in universal algebras," Annals of Fuzzy Mathematics and Informatics, vol. 17, no. 1, pp. 31-39, 2019.
[5] B. A. Alaba and G. M. Addis, " $L$-fuzzy prime ideals in universal algebras," Advances in Fuzzy Systems, vol. 2019, Article ID 5925036, 7 pages, 2019.

[6] B. A. Alaba and T. G. Alemayehu, "Closure fuzzy ideals of MSalgebras," Annals of Fuzzy Mathematics and Informatics, vol. 16, pp. 247-260, 2018.

[7] B. A. Alaba and W. Z. Norahun, "Fuzzy ideals and fuzzy filters of pseudo-complemented semilattices," Advances in Fuzzy Systems, vol. 2019, Article ID 4263923, 13 pages, 2019.

[8] U. M. Swamy and D. V. Raju, "Fuzzy ideals and congruences of lattices," Fuzzy Sets and Systems, vol. 95, no. 2, pp. 249-253, 1998.

[9] B. A. Alaba, M. A. Taye, and D. A. Engidaw, " $L$-fuzzy ideals of a poset," Annals of Fuzzy Mathematics and Informatics, vol. 16, no. 3, pp. 285-299, 2018.

[10] U. M. Swamy and D. V. Raju, "Algebraic fuzzy systems," Fuzzy Sets and Systems, vol. 41, no. 2, pp. 187-194, 1991.

[11] U. M. Swamy and D. Viswanadha Raju, "Irreducibility in algebraic fuzzy systems," Fuzzy Sets and Systems, vol. 41, no. 2, pp. 233-241, 1991.

[12] S. Mahmood, M. Ulrazaq, S. Abdul-Ghani, and A. Al-Musawi, " $\sigma$ algebra and $\sigma$ baire in fuzzy soft setting," Advances in Fuzzy Systems, vol. 2018, p. 10, Article ID 5731682, 2018.

[13] S. Mahmood, "Dissimilarity fuzzy soft points and their applications," Fuzzy Information and Engineering, vol. 8, no. 3, pp. 281-294, 2016.

[14] S. Mahmood, "Decision making using algebraic operations on soft effect matrix as new category of similarity measures and study their application in medical diagnosis problems," Journal of Intelligent and Fuzzy Systems, vol. 37, no. 2, pp. 1865-1877, 2019.

[15] J. A. Goguen, "L-fuzzy sets," Journal of Mathematical Analysis and Applications, vol. 18, no. 1, pp. 145-174, 1967.

[16] U. M. Swamy and K. L. N. Swamy, "Fuzzy prime ideals of rings," Journal of Mathematical Analysis and Applications, vol. 134, no. 1, pp. 94-103, 1988.

[17] A. Diego, "Sur les algèbres de Hilbert, Collection de Logique mathematique. Series A," in Fuzzy Sets and Systems, Hermann, Ed., vol. 44, pp. 127-138, Kluwer Academic, Boston, London, 1966.

[18] D. Busneag, "A note on deductive systems of a Hilbert algebra," Kobe Journal of Mathematics, vol. 2, p. 2935, 1985.

[19] D. Busneag, "Hilbert algebras of fractions and maximal Hilbert algebras of quotients," Kobe Journal of Mathematics, vol. 5, p. 161172, 1988.

[20] Y. B. Jun and S. M. Hong, "Fuzzy deductive systems in Hilbert algebras," Indian Journal of Pure and Applied Mathematics, vol. 27, no. 2, pp. 141-151, 1996.

[21] Y. B. Jun, "Extensions of fuzzy deductive systems in Hilbert algebras," Fuzzy Sets and Systems, vol. 79, no. 2, pp. 263-265, 1996.

[22] I. Chajda, "The lattice of deductive systems on Hilbert algebras," Southeast Asian Bulletin of Mathematics, vol. 26, no. 1, pp. 21-26, 2003.

[23] B. B. N. Koguep and C. Lele, "On fuzzy prime ideals of lattices," SJPAM, vol. 3, pp. 1-11, 2008.

[24] V. Murali, "Fuzzy congruence relations," Fuzzy Sets and Systems, vol. 41, no. 3, pp. 359-369, 1991. 